\title{
RESOLVE's Field Demonstration on Mauna Kea, Hawaii 2010
}

\author{
Janine Captain ${ }^{1}$, Jacqueline Quinn ${ }^{2}$ and Thomas Moss ${ }^{3}$ \\ National Aeronautics and Space Administration, Kennedy Space Center, FL, 32899 \\ Kyle Weis ${ }^{4}$ \\ ASRC Aerospace Corporation, Kennedy Space Center, FL, 32899
}

\begin{abstract}
In cooperation with the Canadian Space Agency, and the Northern Centre for Advanced Technology, Inc., NASA has undertaken the In-Situ Resource Utilization (ISRU) project called RESOLVE (Regolith and Environment Science \& Oxygen and Lunar Volatile Extraction). This project is an Earth-based lunar precursor demonstration of a system that could be sent to explore permanently shadowed polar lunar craters, where it would drill into regolith, quantify the volatiles that are present, and extract oxygen by hydrogen reduction of iron oxides. The resulting water could be electrolyzed into oxygen to support exploration and hydrogen, which would be recycled through the process. The RESOLVE chemical processing system was mounted on a Canadian Space Agency mobility chasis and successfully demonstrated on Hawaii's Mauna Kea volcano in February 2010. The RESOLVE unit is the initial prototype of a robotic prospecting mission to the Moon. RESOLVE is designed to go to the poles of the Moon to "ground truth" the form and concentration of the hydrogen/water/hydroxyl that has been seen from orbit $\left(\mathrm{M}^{3}\right.$, Lunar Prospector and LRO) and to test technologies to extract oxygen from the lunar regolith. RESOLVE has the ability to capture a one-meter core sample of lunar regolith and heat it to determine the volatiles that may be released and then demonstrate the production of oxygen from minerals found in the regolith. The RESOLVE project, which is led by $\mathrm{KSC}$, is a multi-center and multi-organizational effort that includes representatives from KSC, JSC, GRC, the Canadian Space Agency, and the Northern Center for Advanced Technology (NORCAT). This paper details the results obtained from four days of lunar analog testing that included gas chromatograph analysis for volatile components, remote control of chemistry and drilling operations via satalite communications, and real-time water quantification using a novel capacitance measurement technique.
\end{abstract}

\section{Introduction}

In order to better understand the evolution of the planets and solar system, research that contributes to the development of new techniques for further space exploration is needed. As NASA continues to explore new frontiers, future human missions will rely on local natural resources (ISRU) to produce any required infrastructure

\footnotetext{
${ }^{1}$ Chemist, Applied Physics Branch, NE-L5, National Aeronautics and Space Administration, Kennedy Space Center, FL, 32899

${ }^{2}$ Environmental Engineer, Surface Systems Branch, NE-S, National Aeronautics and Space Administration, Kennedy Space Center, FL, 32899

${ }^{3}$ Senior Computer Engineer, Applied Physics Branch, NE-L5, National Aeronautics and Space Administration, Kennedy Space Center, FL, 32899

${ }^{4}$ Chemist, Department Name, ASRC-15, ASRC Aerospace Corporation, Kennedy Space Center, FL, 32899
} 
(habitats, fuel, oxygen and other life support materials). Analog field testing locations have been developed and utilized to test equipment and instrumentation that are being developed in support of the Exploration initiative.

One such instrumentation package developed to support ISRU is RESOLVE; a drilling and chemistry plant that can be packaged onto a medium-sized rover. RESOLVE's chemistry plant has the ability to analyze soil for volatile components using an onboard gas chromatograph prior reducing the soil at high temperatures in the presence of hydrogen to generate water (also called hydrogen reduction). RESOLVE, which stands for Regolith and Environment Science \& Oxygen and Lunar Volatile Extraction, was built to perform lunar analog activities that would simulate flight hardware that could be sent to a permanently shadowed, polar lunar crater to determine the form of the hydrogen indicated by lunar orbiters as well as extract oxygen using hydrogen reduction.

The RESOLVE Prototype consists of EBRC (Excavation and Bulk Regolith Characterization, i.e. a Drill and a Crusher), the Regolith Volatile Characterization (RVC) subsystem (i.e. a reactor and a gas chromatograph), the Lunar Water Resource Demonstration (LWRD) subsystem, and the Regolith Oxygen Extraction (ROE) subsystem. The RESOLVE Prototype processing module can be mounted onto any mobility platform that would accept its current mass and volume configuration. RESOLVE's capabilities include drilling one meter into soil, taking core samples, crushing them into $1 \mathrm{~mm}$ particles, delivering them to the Reactor, heating one quarter-meter core sample at a time and driving off volatiles, analyzing the volatiles, capturing the water and hydrogen evolved, and extracting oxygen by hydrogen reduction.

This paper details the RESOLVE system, the modifications and its performance in concert with a Canadian Space Agency (CSA)-lead field campaign at a site on the Mauna Kea volcano on the Big Island of Hawaii (altitude of $2740 \mathrm{~m}(9000 \mathrm{ft})$ ) in February, 2010. In the 1960s, Apollo astronauts prepared for their missions on Mauna Kea and declared those sites to be the most realistic in terms of the terrain in the lunar environment they encountered. The site on Mauna Kea for the RESOLVE demonstration has already been prepared by PISCES (Pacific International Space Center for Exploration Systems), a center at the University of Hawaii - Hilo. A new mobility platform was provided by CSA and some modifications to the RESOLVE plant were required. This work included minimal reconfiguration to be accepted on a new mobility platform, laboratory retesting of the current configuration, and participation in the February, 2010 field campaign where volatiles and water analyses would be performed. This field activity allowed RESOLVE to be tested on CSA-provided mobility platforms that have the capability to traverse along steeper crater slopes.

\section{Background of RESOLVE}

When mankind returns to the moon, there will be one aspect of the architecture that will totally change how we explore the solar system. For the first time in space exploration, we will take the initial steps towards breaking our reliance on Earth-supplied consumables by extracting resources from planetary bodies. Our first efforts in this area, known as In Situ Resource Utilization (ISRU), will be directed at extracting some of the abundant oxygen found in the lunar regolith. But the "holy grail" of lunar ISRU will be finding an exploitable source of lunar hydrogen. If we can find a source of hydrogen that can be reasonably extracted from the regolith, it would provide a foundation for true independence from Earth consumables. With in-situ hydrogen and oxygen (and/or water) we can produce many of the major consumables needed to travel to and operate on a sustainable lunar outpost. We would have water to drink, oxygen to breath, and rocket propellants and fuel cell reagents to enable extended access and operations across the moon. These items make up a huge percentage of the mass launched from the Earth and the consumables delivered to a lunar outpost. Producing them in situ would also significantly reduce the cost of operating a lunar outpost while increasing the payload capability for other lunar exploration objectives, such as science.

The Lunar Prospector mission found evidence of elevated hydrogen at the lunar poles, and measurements made at these locations from the Clementine mission bistatic radar have been interpreted as correlating to water/ice concentrations ${ }^{1-3}$. At the South Pole, there is reasonably strong correlation between the elevated areas of hydrogen and permanently shadowed craters. More recently, LCROSS (Lunar Crater Observation and Sensing Satellite) and $\mathrm{M}^{3}$ (Moon Mapping Mission) ${ }^{4}$ found strong evidence of water ice only centimenters below of the surface in the shadowed regions of the poles. However, the only way to truly answer the question of the type and availability of the hydrogen signal detected by these more recent missions is to go to the lunar poles and make direct measurements of the regolith. With this in mind, NASA initiated development of an experiment package named RESOLVE (Regolith \& Environment Science and Oxygen \& Lunar Volatile Extraction) that could be flown to the rim or into a permanently shadowed crater to answer the questions surrounding elevated hydrogen at the lunar poles. RESOLVE is a drilling and miniature chemistry plant packaged onto a medium-sized rover that analyzes collected soil for volatile components prior to heating the soil and reducing it at high temperatures in the presence of hydrogen to 
produce water. The work performed in 2010 tested only the volatiles characterization capabilities of this autonomous instrument during a Canadian Space Agency-lead activity on Mauna Kea, Hawaii.

The presence of a hydrogen and water signal on the lunar surface has led to a desire for a mission to provide ground truth data to support the spectroscopic data. The requirements of this instrument package include ability to clearly distinguish between hydrogen and water as well as quantify low levels of those species. The RESOLVE Regolith Volatile Characterization (RVC) system was designed to evolve and analyze volatile species from regolith samples. The system successfully characterized volatile samples in a previous field test in 2008 and in a laboratory setting. Previous field test results analyzed samples doped with a low level of water ( $\sim 1 \%$ by weight) in the field. One of the goals of this field test was to demonstrate the detection of low levels of hydrogen and water evolved during heating of a tephra sample. There were several additional goals that evolved during the integration period leading to the field test. These included remote command/control of the system from KSC through the CSA provided satellite link, chemical processing while roving, and operation from battery power on a tandem unit to eliminate dependency on a generator.

\section{RESOLVE Modifications and Preparations for 2010 Field Test}

\section{A. Hardware and site configuration for 2010 Field Test}

The RESOLVE system reconfiguration conducted for the 2010 Hawaii Analog Field Test included the following changes; power change to accommodate operation from a battery source, electronics rack repackaging for installation onto a rover, integration to Canadian Juno tandem rover, and transition to Wi-Fi 802.11 communication.

A.
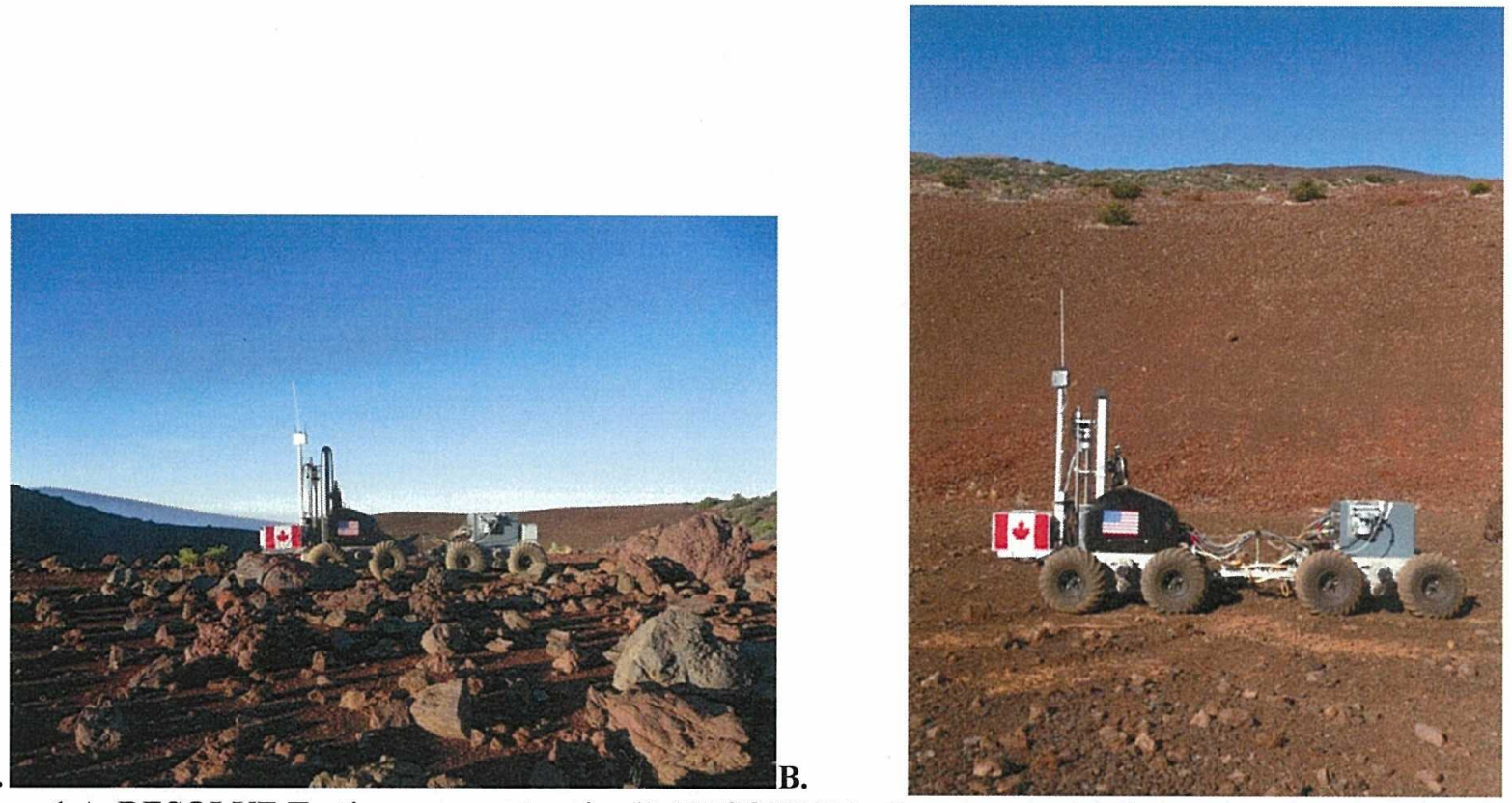

Figure 1 A. RESOLVE Testing on a crater rim, B. RESOVLE testing on a crater floor.

The water capture system in the LWRD system was replaced with the capacitance sensor water capture system from the ROE system, and the hydrogen capture system was removed due to time limitations for the field test.

During the 2008 Hawaii integrated field test, the RESOLVE system was powered using a generator producing 220 VAC. This voltage was routed through a power supply providing $28 \mathrm{VDC}$ to the component systems of the RESOLVE electronics package. As a step forward to this year's activities, there was a desire to run the system from batteries and decrease generator dependency in the field test. A component investigation was conducted to determine the impact of transitioning from 28VDC power to the lower 24VDC power with droop supplied by the batteries. It was found that all but a few components were capable of operating at the lower voltage. A small secondary de to dc converter was put in line with this system to boost the power to 28VDC for these few components. There were two components that required 110VAC for operation; therefore an inverter was selected and installed to provide power to those components. 
During system checkout and integrated testing, there was a need to operate in the laboratory from standard wall power. To allow for this dual operation, the primary power supply was retained in the system and adjusted to supply $24 \mathrm{VDC}$ rather than the $28 \mathrm{VDC}$. To protect against powering the battery hookup via the power supply, or back powering the supply, a pair of relays controlled by a keyed switch were installed. These relays also provided a mechanism for a safety E-stop which was installed in series with the keyed switch.

The electronics rack repackaging was dictated by a desire to be mobile and mount on the tandem rover unit (Fig. 1). This modification allowed increased mobility for the system and autonomy compared to the previous field test. A smaller 32" tall rack was identified and retasked to house the electronics package, power supply, inverter, vacuum pump and source switching components. These components were rewired into the smaller rack with a significant volume savings. Brackets were installed on this rack to interface with the Canadian Juno Rover.

Complementing these activities was the chemistry plant integration to the lead rover of the tandem unit. Because the system was designed to be modular and simply pin mount in place, this was quite simple once the rover brackets where in place. A fiberglass body shell was made for the unit for thermal and aesthetic considerations.

As an alternative to mounting two radio modems on the Juno rover (one for the rover/drill and the other for the chemistry plant) as was done in the past, a broad Wi-Fi mesh was supplied by NORCAT that would allow sufficient bandwidth for both systems to communicate over a single link. To this end a wireless switch with multiple Ethernet ports was mounted on the rover. This allowed communication to be established simply plugging the system into this switch, thus attaching the RESOLVE Master Evenet Controller (MEC) to the field site network.

\section{B. Control System and Software Modifications}

To mitigate communication issues caused by an overloaded National Instruments (NI) compact RIO (cRIO) embedded controller, a new cRIO was procured with increased processing power. The new cRIO required a complete NI software toolkit upgrade and a recompile of the FPGA code. The upgraded LabVIEW had a significant bug that would not allow the new cRIO to operate. Online research indicated a coding technique that would work around the software bug and allow normal cRIO operation. The new cRIO operated much more stably and also provided performance margin for $\mathrm{cRIO}$ code modifications.

Numerous cRIO code modifications were added to improve usability and to provide software solutions to hardware problems. During the previous analog demonstration, there had been an intermittent issue with a specific communications protocol. To ensure proper operation, prompts had been added to pause software sequencing and to notify the operator whenever a critical OPC command was sent. With the more stable system, code was added to the cRIO to allow the operator to turn off the OPC prompts during non-critical testing.

Another usability improvement was related to limit monitoring. The software system monitored analog measurements and would notify the operator whenever an analog measurement violated its preset limits. If the operator did not respond to the prompt, the software would safe the system and then shut it down. There were many instances during lab operation when the system was not completely configured leaving analog measurements unpowered and completely violating their limits. These unpowered measurements would cause numerous prompts and possibly system shutdown. The cRIO software was modified to allow the operator to bypass analog measurement monitoring for non-critical testing.

One hardware issue that was mitigated through software was the loss of a critical thermocouple which was used to control the heating of the reactor's auger. There was not a redundant measurement so the most representative working thermocouple was used as the auger heater control point. Since the auger temperature control was very sensitive, it was necessary to add a duty cycle control to the auger heater to keep it from overheating.

Another hardware issue was addressed through a software change during the analog field test. There are ball valves for allowing regolith samples to be fed into and released from the reactor. During the field demonstration, the valves did not work properly. The cRIO code was modified to add the capability to incrementally "bump" the valves into proper positions in order to allow the testing to continue.

The HMI LabVIEW code, running on a remote laptop, also had some changes implemented. The display was modified to reflect the chemical plant hardware changes that took place since the 2008 Lunar Analog Demonstration. The data logging code was also updated to reflect those hardware changes. One other application was created to allow the operator to save an entire Process with its Sequences each containing its associated list of tasks to a spreadsheet file. This allowed the operators verify that the process was indeed constructed as intended.

\section{Laboratory testing of GC}

Two MicroSAM gas chromatograph systems were modified for the volatile analysis, providing a main and backup system for the field test. Both gas chromatograph systems were outfitted with a lightweight case, neon carrier gas, heated sample transfer lines, and a heated sample loop. The backup system was also modified with a 
second oven and re-plumbed to allow the mol-sieve column to operate outside of the module with separate heater control.

The operation of the $\mathrm{GC}$ was optimized for $\mathrm{H}_{2}, \mathrm{He}$, and water detection. Other compounds that could be quantified were $\mathrm{N}_{2}, \mathrm{O}_{2}$ or $\mathrm{Ar}, \mathrm{CO}_{2}, \mathrm{CO}, \mathrm{CH}_{4}$, and $\mathrm{H}_{2} \mathrm{~S}$. The sample in the field introduced to the $\mathrm{GC}$ was controlled because of the specific scientific demonstration goals of this field test. For this reason, only a select set of gases were used to create calibration curves. If any uncalibrated species were observed at the field test site, calibration curves could be run upon completion of the field testing. The gases used for calibration included $\mathrm{N}_{2}$, $\mathrm{O}_{2}, \mathrm{CO}_{2}, \mathrm{Ar}, \mathrm{H}_{2}$ and $\mathrm{He}$. The separation of water, $\mathrm{CO}_{2}$ and inert species was performed on a Porabond$\mathrm{Q}$ column, with a heart cut to separate the inert species on a mol-sieve column.

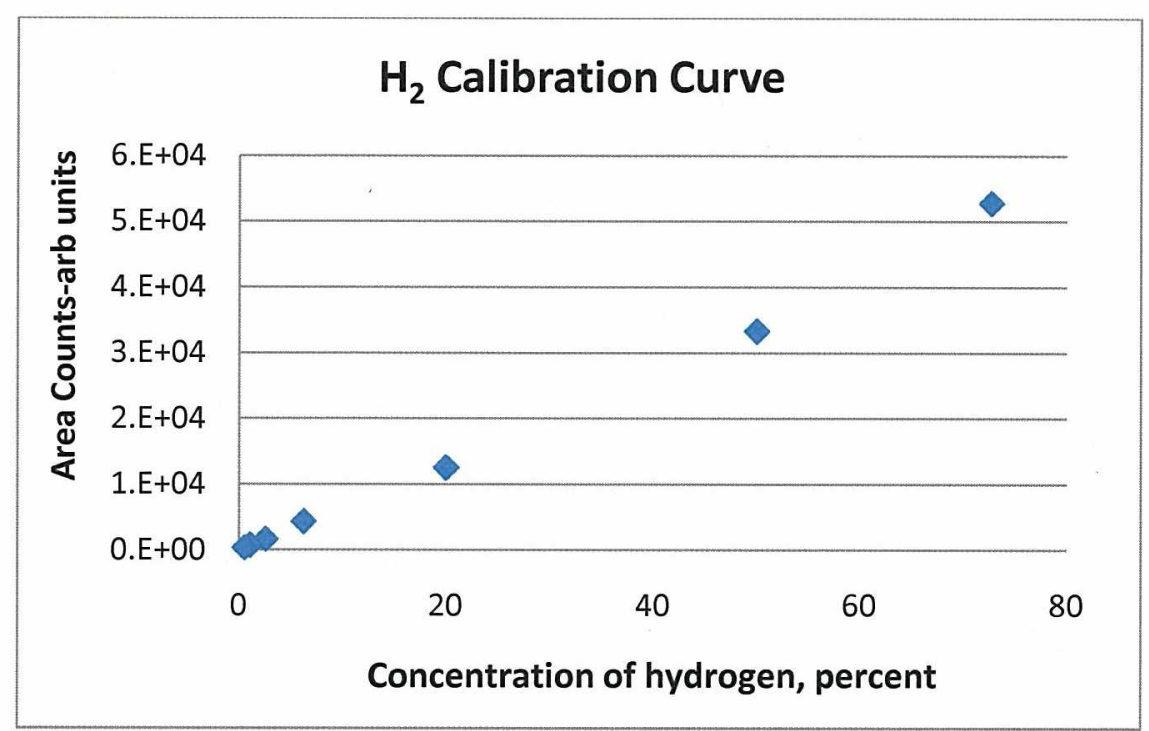

Figure 2. Hydrogen calibration curve for $300 \mathrm{~ms}$ injection For the calibration runs, the sample injection times used were 100,300 , and $600 \mathrm{~ms}$. The increasing time corresponds to a larger sample amount injected. Longer injection times allowed for increased limit of detection for compounds present in low concentrations while the smaller injection times were used to prevent overloading of the column. The calibration curve for hydrogen with a $300 \mathrm{~ms}$ injection time is shown in Fig. 2.

Calibration of water at high concentrations has been and continues to be a challenge for GC measurements. Water is extremely susceptible to condensation and even at elevated temperatures can coat nearly all surfaces. For this reason, the determination of the amount of water in the reactor was performed indirectly. All of the other species in the reactor were calibrated and could be quantified with calibration curves. The remaining percentage not accounted for by the calibrated species was attributed to water. This method was used because all the species, with the exception of water, were calibrated in the GC sample. Future work is underway on a high concentration water vapor generator.

GC provides the composition of the gas sample where the area of the peak corresponds to the percentage of that component in the sample. Because the sample injected into the GC is compressed prior to injection onto the column, the system is not sensitive to the pressure of the reactor provided there is sufficient pressure to purge the sample loop of the GC. This means a 50/50 mixture of hydrogen and water in the reactor at 50psia will give the same spectra and peak areas as a 50/50 mixture of hydrogen and water at 20psia. To calculate the amount of volatiles, the total amount of gas in the reactor must be calculated. This is done using the ideal gas law. The pressure of the reactor is measured with an absolute pressure transducer, the volume of the reactor is known, and the temperature used is an average temperature of five thermocouples on the reactor body. The GC analysis which provides the percentage of each component is multiplied by the total number of moles to obtain the moles of each component present in the reactor.

\section{Water and Hydrogen doping of sample}

Water doping of the soil was performed by two methods. The first method was to simply expose the dried and sieved tephra sample to atmospheric conditions. The tephra absorbs a small amount of water from the moisture in the atmosphere, typically coming to a water content of about $1 \%$ by weight. The water content of samples treated in this manner was measured up to $150 \mathrm{C}$ in open air with a laboratory soil moisture analyzer. Higher water concentrations of water were achieved by doping a small amount of tephra with liquid water and adding the doped sample to the reactor. In this manner up to an additional 0.5 grams of water was added to the reactor.

One of the scientific goals of this test was to show hydrogen evolution with increasing temperature. Several methods of doping the reactor with hydrogen were explored in the laboratory. The method chosen for this field test 
was the use of a metal hydride. Metal hydrides are typically used to generate and store hydrogen, however most metal hydrides are sensitive to air. For our specific application, the desired characteristics of the metal hydride included the storage hydrogen at room temperature, allowance of a safe transfer in air to the reactor, and the release hydrogen as it was heated to $150 \mathrm{C}$. Ideally the metal hydride would have a low vapor pressure at room temperature and a significant vapor pressure at $150 \mathrm{C}$, releasing all of the hydrogen in the sample. The hydride chosen was Hystor 207, a lanthanum nickel aluminum metal hydride with a vapor pressure of 0.42 atmospheres at $25 \mathrm{C}$ and 42 atmospheres at $175 \mathrm{C}$. This metal hydride had the desirable range of vapor pressure however it was corroded by oxygen and water. The challenge of using this metal hydride in the air safely was overcome by passivating the surface in a controlled environment. While this procedure decreased the overall hydrogen capacity of the metal hydride, it allowed enough hydrogen to be retained in the sample for evolution upon heating in the reactor. To prevent a flammable mixture of gas in the reactor during heating, the reactor was purged with argon prior to heating.

Several batches of metal hydride were prepared and tested in the lab to verify the evolution of hydrogen after passivating the sample. Initial samples were tested in a custom purged reactor and analyzed with the RESOLVE GC system. Testing in the lab indicated the passivated sample could be used for several days and reliably evolve hydrogen upon heating. Due to many unknowns during the activation and passivation procedures (such as surface area, elapsed time prior to heating in the reactor), the amount of metal hydride added to the reactor was not directly related to the amount of hydrogen evolved during the heating profile. The laboratory experiments indicated that the metal hydride would provide hydrogen within the detection limit that was desired, however the exact amount of evolved hydrogen could not be calculated.

\section{E. Laboratory testing of Capacitance Sensors}

Capacitance based level sensors are commonly used in commercial liquid level applications. In this application of capacitance measurement, the hygroscopic nature of the desiccant combined with the flow characteristics ensure that the moisture will fill the desiccant in a fashion similar to a tank being filled from bottom to top. This allows the capacitor to act in a similar fashion to the standard liquid level sensor. The dielectric constant of the Moisture Gone desiccant was measured to be approximately 1.5 when dry and 32.3 when totally wet. This ensures that the sensor will have a strong capacitance change when moisture is introduced to the bed.

The capacitance probe consisted of two electrodes. The first electrode was a 1/8" diameter stainless steel rod inserted down the middle of the water bed. On one end, the rod was seated in a ceramic flange. The other end was fitted with a sleeve and protruded out the end of the bed. The second electrode was the outer casing of the water bed. Therefore, the field lines ran radially between the center rod and the bed casing. The MoistureGone desiccant filled the annular space between the inner and outer electrodes.

A pair of desiccant beds with capacitance measurement capabilities were built and utilized for the hydrogen reduction operations during the 2008 field

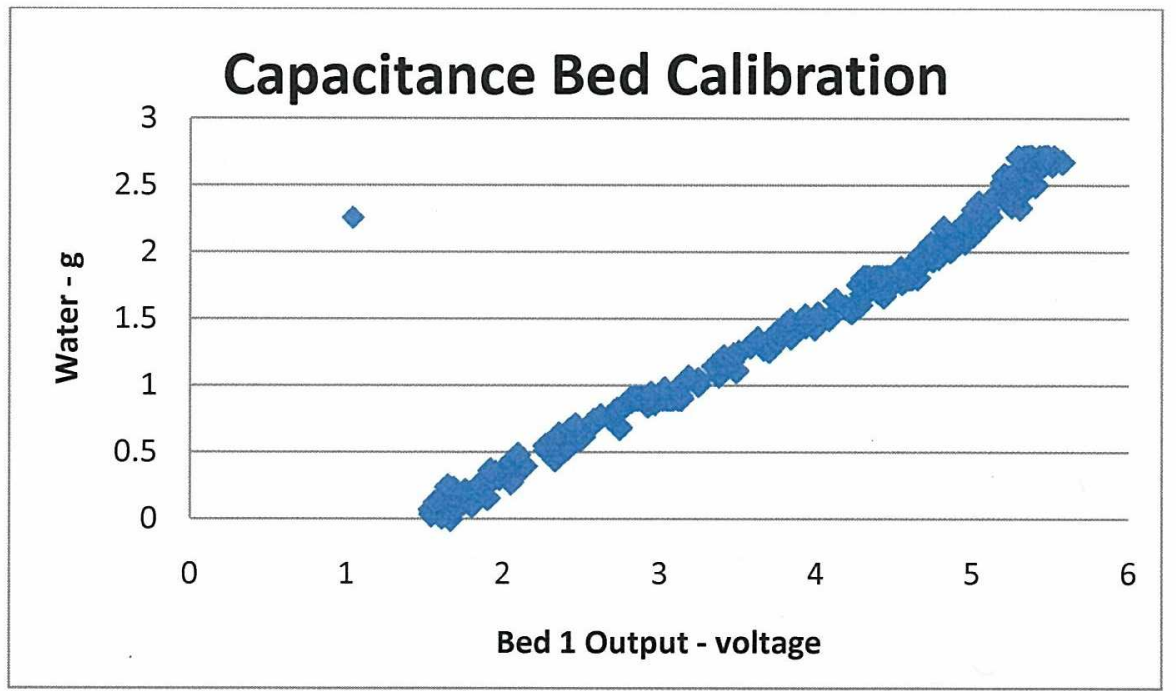

Fig 3. Calibration curve of capacitance sensor in water capture bed

test. It was demonstrated that the capacitance beds could detect the water being generated by the oxygen production system. Each bed was designed to measure up to $3 \mathrm{~g}$ of water with an accuracy of $1 \%$.

These capacitance beds were subsequently retrofitted to quantify the water released in the volatile characterization processes for the 2010 field test. An independent thermal control loop was added to the beds to allow the beds to be desorbed in place. MLI (multi layer insulation) was also added to the beds to avoid over heating the $130^{\circ} \mathrm{C}$ box components during water desorption. 
To calibrate the capacitance water beds, the dried beds were controlled to a temperature of $130^{\circ} \mathrm{C}$ while a flow of humid air $\left(56 \%\right.$ relative humidity $(\mathrm{RH})$ at $\left.22^{\circ} \mathrm{C}\right)$ was passed through the beds. The total amount of water added to the beds was calculated from the RH and flow rate of gas (Fig. 3).

\section{F. Integrated laboratory tests of the RESOLVE system}

Three integrated samples were analyzed in the laboratory setting prior to the field test of the RESOLVE system. The tephra used in the lab tests was dried at $130 \mathrm{C}$ for several days prior to use. Tephra samples were cooled, weighed, and a small portion of the tephra sample was dosed with water and mixed with metal hydride prior to introduction into the reactor. In addition to water and hydrogen evolution during heating, carbon dioxide was also evolving from the sample with increasing temperature.

The first integrated test on Jan $5^{\text {th }}$ (5-Jan) consisted of $83.3 \mathrm{~g}$ tephra, $3.08 \mathrm{~g}$ metal hydride, and $0.25 \mathrm{~mL}$ water. The heating profile to $150 \mathrm{C}$ took 46 minutes with a maximum pressure of $28 \mathrm{psia}$ and a final pressure of $25 \mathrm{psia}$ prior to transfer to the surge tank. The initial pressure of the argon purge in the system was $\sim 20 \mathrm{psia}$. The final gas composition in the reactor prior to transfer to the surge tank was $0.24 \mathrm{mg}$ hydrogen, $23.6 \mathrm{mg}$ water, and $8.7 \mathrm{mg}$ of carbon dioxide in an argon atmosphere. The second integrated test (12-Jan A) consisted of $80.2 \mathrm{~g}$ tephra, $4.08 \mathrm{~g}$ metal hydride, and $0.2 \mathrm{~mL}$ water. The 42 minute heating profile evolved about half of the amount of water (12mg) as the first integrated lab test, a similar amount of carbon dioxide $(5.8 \mathrm{mg})$, but significantly more hydrogen $(10 \mathrm{mg})$.

The final integrated test in the lab (12-Jan B) was composed of $80.3 \mathrm{~g}$ tephra, $3.15 \mathrm{~g}$ metal hydride, and $0.5 \mathrm{~mL}$ water. This integrated test took 45 minutes to reach the $150 \mathrm{C}$ set point and transfer the sample to the surge tank. The makeup of the evolved gases in the reactor prior to the first reactor transfer was $7.6 \mathrm{mg}$ hydrogen, $53 \mathrm{mg}$ water, and $13.8 \mathrm{mg}$ carbon dioxide. This test was unique because after the first transfer to the surge tank, there was sufficient pressure in the reactor to continue to sample with the GC and transfer a second sample to the surge tank.

\section{Field Test Activities}

\section{A. Integrated Pre-deployment Test in Sudbury Canada}

A pre-deployment integrated test was held in Sudbury in October 2009. This test allowed for several of the modifications to the RESOLVE system to be checked out prior to the field test in Hawaii. The main tests included operating from a battery source to determine lifetime, droop, and any hot swap capabilities, a fit check on the Juno tandem rover, and communication tests for remote command and control through CSA's ExDOC facility to KSC.

This integration test was successful for testing many of the additional goals that the team hoped to achieve during the field test. The fit test for both the chemistry plant and the miniaturized electronics rack onto the tandem rover assembly was successful and demonstrated the roving capability of the loaded unit on several different slopes in the local quarry in Sudbury used for the pre-deployment testing.

The system was powered by the battery packs in the rails of the rover and an auxiliary set of battery packs on the rover unit that carried the electronics. The battery voltage started at $24.3 \mathrm{VDC}$, and over 2 days of operation, the voltage dropped to $22.3 \mathrm{VDC}$. The system successfully operated at this voltage at which point a hot swap of the batteries was attempted. The four battery packs were each changed out sequentially while RESOLVE continued to operate, demonstrating a successful battery change out in the field during operations.

Also successfully demonstrated in this pre-deployment test was remote command and control through the CSA ExDOC facility to the Kennedy Space Center. The ExDOC facility at CSA has been used for command and control in other field test campaigns and is modeled after the control center used to control the CSA provided hardware on the ISS. Although this test did not utilize the satellite link that would be used for communication at the field test site on Mauna Kea, the communication protocol through the firewalls at KSC and CSA were successfully negotiated. Operators at KSC successfully performed several manual and automated sequences while monitoring the data. A time delay of 15-30 seconds between the commands and execution was typical during remote operations through ExDOC.

\section{B. Volatile analysis of samples at Field Test Site}

Sample cores were prepared in the field with sieved and dried soil for the RESOLVE system. The sample transferred to the reactor was then doped with the water bearing tephra and metal hydride and the system was purged with argon to provide an inert atmosphere. The initial pressure of the argon purge in the reactor was $\sim 12 \mathrm{psia}$ in the field tests compared to $\sim 20$ psia in the lab tests. The thermal profile of the reactor was also different in the field due to the loss of the core heater thermocouple and a heater on the bottom of the reactor body. 
The sample from $2 / 3$ was processed at the second sampling site which was located on top of the ridge. This sample was processed while the drill was operating at the site collecting the next sample. The total weight of the sample in the reactor was $82.3 \mathrm{~g}$, which included $10 \mathrm{~g}$ of metal hydride and $0.3 \mathrm{~mL}$ of water. As the sample was heated, hydrogen, carbon dioxide and water evolved with increasing temperature as shown in Figs.4-5. Prior to the transfer of the sample from the reactor to the surge tank, the gas composition in the reactor was $39.4 \%$ hydrogen, $6.9 \%$ argon, $9.5 \%$ carbon dioxide, and $44 \%$ water. The pressure at the time of the final GC run was 13.8 psia with an average temperature of $155 \mathrm{C}$ after 95 minutes of heating. These values were converted to masses assuming the ideal gas law using the pressure, volume and temperature of the reactor. The mass of hydrogen, water and carbon dioxide were $0.00369 \mathrm{~g}, 0.03710 \mathrm{~g}$ and $0.01959 \mathrm{~g}$ respectively. These results show that the RESOLVE gas analysis system is able to analyze very low weight percents of volatiles in tephra, well below $0.1 \%$ by weight. Data from the remaining three samples which were analyzed at sampling sites three and four in the crater floor (4-Feb - 5-Feb) have similar low levels of volatiles, ranging from $6 \times 10^{-5}$ to $0.005 \mathrm{wt} \%$ hydrogen and 0.025 to $0.031 \mathrm{wt} \%$ water.

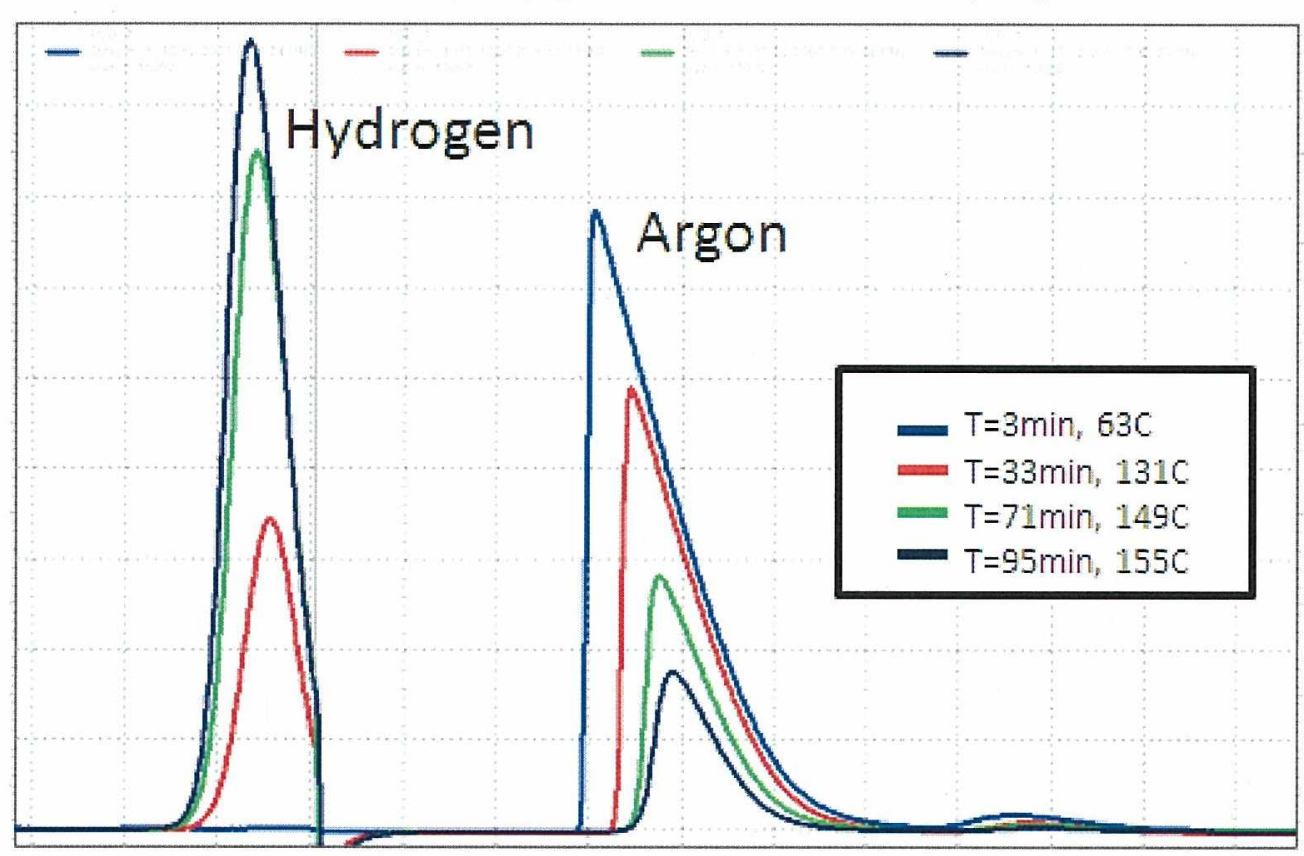

Fig 4 TCD1 spectra, data from $2 / 3 / 2010$ showing decreasing concentration of argon and increasing concentration of hydrogen with increasing reactor temperature
While the water detected in the reactor shows the ability for the system to quantify low levels of water in the tephra samples, the water abundance does not directly correlate with the amount of water added to the reactor.

This discrepancy is due to a number of factors. The first is the fact that the water is bound to the

tephra which is very slow to release this water. Water can become bound in hydrated minerals causing the vapor pressure of water above the tephra to be much lower than that of unbound water, resulting in a low vapor concentration of water. The second factor is the leak rate of the reactor allowed some water to escape during the heating process. The requirement to purge the sample to provide an inert atmosphere also affected the water content of the sample. Flowing dry argon over the sample for 10 minutes most likely stripped the loosely bound water in the tephra sample. Finally, the high surface area of the tephra and reactor components provided a huge number of adsorption sites for water, again decreasing the water vapor concentration in the reactor. The water in the gas phase of the reactor varied from $12 \mathrm{mg}$ to $66 \mathrm{mg}$ between the laboratory and field testing of the integrated system. 
C.

Capacitance

Mesurements

After the

reactor reached

a temperature

of $150^{\circ} \mathrm{C}$ and a

final $\mathrm{GC}$

sample was

taken, a valve

was opened

between the

evacuated surge

tank and the

reactor. This

transferred

most of the

volatiles from

the reactor to

the surge tank.

After the

reactor was

isolated from

the surge tank,

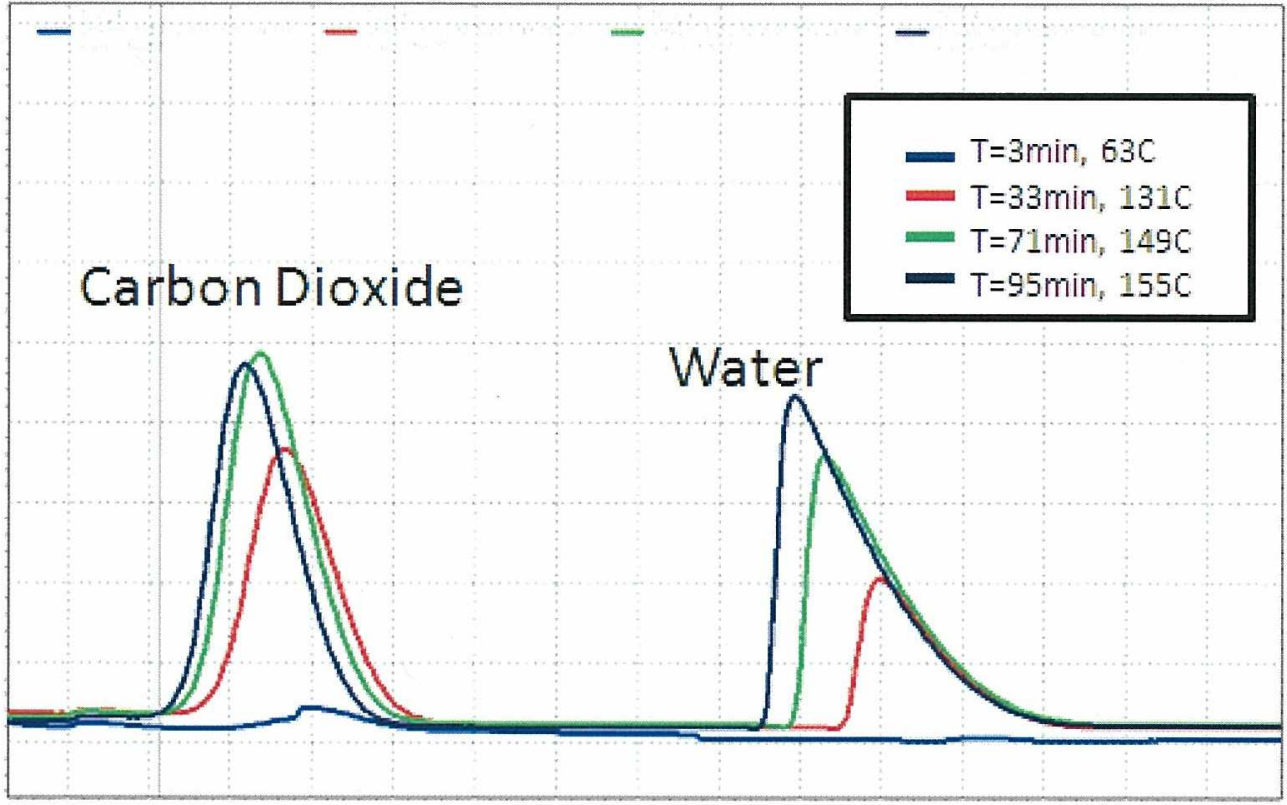

Fig 5 TCD4 spectra, data from 2/3/2010 showing increasing carbon dioxide and water concentrations with increasing reactor temperature

the valves to

the desiccant beds were opened and the circulating pump was run for 3 minutes. During previous testing this time was found to sufficiently dry the gas circulating in the LWRD system.

In theory, the water should be captured in the first bed similar to a tank filling with water. This allows the theory of a level sensor based on capacitance to be used for the data analysis of the system. While at a gross level this behavior may be true, there is most likely some channeling and redistribution of the water vapor within the adsorption beds over the duration of the field test. These two phenomenon can lead to errant measurements, especially when attempting to measure small quantities of water. Each capacitance bed was designed to capture and measure $3 \mathrm{~g}$ of water with $1 \%$ accuracy. The additional noise from the signal conditioning led to a decreased level of accuracy. The capacitance data was fairly noisy as seen during the calibration of the system. The amount of water measured as well as the standard deviation within these data

\begin{tabular}{|l|l|l|l|}
\hline & & Water $(\mathrm{g})$ & Standard Deviation \\
\hline Feb 3 2010 & & 0.01855 & 0.0305 \\
\hline Feb 4 2010 & & 0.01 & 0.0251 \\
\hline Feb 5 2010 & run1 & 0.0513 & 0.0483 \\
\hline Feb 5 2010 & run2 & 0.0006 & 0.0327 \\
\hline
\end{tabular}

Table 1 Amount of water measured by capacitance sensor and the standard deviation within the data sets used to calculate those values

sets is shown in Table 1. For the majority of the data points, the standard deviation within the data set is larger than the amount of water calculated. The low levels of water vapor produced in the oven did not provide sufficient sample to reach the detection limit of the capacitance sensor in the water capture beds. The increase in temperature may have affected the way in which the water was distributed in the bed during adsorption. The higher temperature most likely provided more opportunity for the redistribution of the water within the capture bed which could affect the measurement. Finally, the noise in the electronics also contributes to the large standard deviation within the system, making the small amount of water transferred to the surge tank impossible for the capacitance sensor to measure. There are several changes to the capacitance sensor that would allow the system to more accurately measure the small concentrations of water seen during this field test. This includes modification to a parallel plate design with a smaller amount of dessicant used. Future work will include optimizing this system for low level water detection.

\section{D.Remote operations from the Field Test site}

An additional capability that was demonstrated during this field test was remote operation of the system over a satellite link. This satellite link was provided by the Canadian Space Agency (CSA). All of the communications 
provided at the field test site (internet, VOIP, streaming video) were sent via a satellite link from the field to ExDOC at CSA. The ExDOC facility is set up to allow users to operate and monitor systems in the field. The communication set up to control the RESOLVE system involved command and control of the system from KSC through the ExDOC satellite link to the field. The connection between KSC and ExDOC was established as a secure connection using NASA Integrated Services Network (NISN).

With LabView as the control software used to operate the RESOLVE system, a remote panel was created that could be used to control the system using a web based internet connection. This method of command and control of the system was tested using the KSC satellite link prior to the deployment to test for communication problems due to the signal delay involved with satellite communication. Testing indicated a significant delay however the system could be succesfully controlled through this link.

In conjunction with the remote panel display of the RESOLVE system with streaming data, a live video stream of four camera views were also streamed back to KSC during the remote operation. This allowed the end user at $\mathrm{KSC}$ to have situational awareness of the surrounding area as well as monitoring the data from the system.

In the field, this communication was set up and preliminarily tested during the system checkout. This allowed the primary users involved in the communication link (RESOLVE field team, ExDOC link, and KSC end user) to ensure the link could be made since this was the first time this configuration was set up and tested. A 15-30 second delay between command issue and response was typical in the field, which the RESOLVE system handled without any problems. The following day at the first sampling site the KSC team took control of the RESOLVE system, initiated several manual commands, monitored the system, and began the automated sequence for sample analysis. This remote operation was performed at the second sampling site and demonstrated for KSC personnel to observe the operations in the field.

\section{Conclusion}

The RESOLVE protype has shown end to end operation of volatile detection at two Field Tests, showing advancements towards flight operation from laboratory investigation. The current system highlights a reuseable reactor that can be used for volatile analysis and oxygen production, GC analysis for quantitation of volatiles, and the ability to capture analytes of interest for ISRU. The low level of detection of water demonstrated in the field test highlight the ability of this system to detect and quantify lunar volatiles. Future work for RESOLVE may include further miniaturization, improvements to dust tolerant seals, addition of a mass spectrometer detector to the GC analysis, and improved thermal characteristics of the system.

\section{Acknowledgments}

The authors would like to thank the MAMMA, DIO, and ISRU programs for funding support. The field test was made possible by the support of the PISCES and SRCan teams. Remote operations were made possible by support from the Canadian Space Agency's ExDOC facility as well as support from NASA-KSC Communication support.

\section{References}

${ }^{1}$ Feldman, W. C., et al., "Fluxes of Fast and Epithermal Neutrons from Lunar Prospector: Evidence for Water Ice at the Lunar Poles," Science 281, p. 1496 (1998)

${ }^{2}$ Feldman, W. C., et al., "Evidence for Water Ice Near the Lunar Poles," J. Geophys. Res. 106, 23231-23251(2001).

${ }^{3}$ Nozette, S., et al., "The Clementine Bi-static Radar Experiment," Science 274, pp. 1495-1498 (1996)

${ }^{4}$ Pieters, C. M. et al., "Character and Spatial Distribution of $\mathrm{OH} / \mathrm{H} 2 \mathrm{O}$ on the Surface of the Moon Seen by M3 on Chandrayaan-1," Science 326, pp. 568-572 (2009)

${ }^{5}$ Lueck, D. E. , Captain, J. E., Gibson, T. L., Peterson, B. V., and Berger, C. M., "Selection, Development and Results for The RESOLVE Regolith Volatiles Characterization Analytical System," CP969, Space Technology and Applications International Forum-STAIF 2008, edited by M. S. El-Genk, American Institute of Physics, Melville, New York, 2008, pp. 149-156. 
${ }^{6}$ Kleinhenz, J., Yuan, Z., Sacksteder, K., and Caruso, J. 2008. "Development of a Reactor for the Extraction of Oxygen and Volatiles from Lunar Regolith". 47th Aerospace Sciences Meeting and Exhibit. American Institute for Aeronautics and Astronautics. Orlando, FL, AIAA-2009-1203

${ }^{7}$ Muscatello A.,Captain J., Lueck D., Quinn J., Gibson T., and Weis K., "Lunar Water Resource Demonstration" 47th Aerospace Sciences Meeting and Exhibit. American Institute for Aeronautics and Astronautics, Orlando, FL, AIAA-2009-1202.

${ }^{8}$ Quinn, J., et al. "Regolith and Environment Science and Oxygen and Lunar Volatile Extraction (RESOLVE) Final Report, March 13, 2009. KSC-KT-10154 